\title{
Incidence of post-operative nausea and vomiting and it's predictors among adult elective surgical patients at Jimma Medical Center, South West Ethiopia
}

Mohammed Suleiman Obsa ( $\square$ msuleiman43@gmail.com )

Wolaita Sodo University https://orcid.org/0000-0001-5078-346X

Dinkisisa Chemeda Edosa

Arsi University College of Health Sciences

Zemenu Muluken Desalegn

Jimma University College of Public Health and Medical Sciences

Nega Desalegn Fanta

Jimma University College of Public Health and Medical Sciences

Sintayehu Mulugeta Tamiru

Mekelle University College of Health Sciences

Gezahegn Tesfaye Mokenin

Jimma University College of Public Health and Medical Sciences

\section{Research}

Keywords: Magnitude, associated factors, post-operative nausea, vomiting, Jimma University Medical center

Posted Date: April 3rd, 2020

DOI: https://doi.org/10.21203/rs.3.rs-19271/v1

License: (c) (i) This work is licensed under a Creative Commons Attribution 4.0 International License. Read Full License 


\section{Abstract}

\section{Background}

Post-operative nausea and vomiting is the most frequent side effect of anesthesia. It affects 20 - 30\% of all post-operative and $70 \%-80 \%$ high risk patients. Consequently, it is one of the most frequently observed adverse events associated with the provision of anesthesia. Thus this study is aimed to assess the incidence and associated factors of post-operative nausea and vomiting.

\section{Methods}

This cross-sectional study was conducted using a consecutive sampling method. Regular supervision and follow up were made. Data was entered in to Epi info version 7 software and transported to SPSS version 20 for analysis. Odd ratio and $95 \%$ confidence interval was computed. The findings of the study were reported using tables, figures and narration. Variables that were found to be candidate ( $p$ value < 0.25 ) on binary logistic regression entered into a multiple logistic regression analysis to identify independent predictors of post-operative nausea and vomiting.

\section{Results}

The results of this study indicated that the incidence of postoperative nausea and vomiting was $27.4 \%$. Output of multiple logistic regression revealed that female sex (AOR $=4.065$ (2.090 - 7.906), history of motion sickness (AOR = 2.836 (1.582 - 5.083), Gynecologic type of surgery (AOR = $3.782(1.156-12.373)$, long duration of anaesthesia (> $60 \mathrm{~min})(A O R=2.974(1.491-5.933)$ and administration of postoperative opioids $(A O R=2.333(1.221-4.457)$ were considered as independent predictors of postoperative nausea and vomiting at $P$ value $<0.05$.

\section{Conclusion}

The present finding has shown that the overall incidence of postoperative nausea and vomiting is high $27.4 \%$ therefore provision of anti-emetic prophylaxis is reccomended.

\section{Background}

Worldwide, more than 310 million major surgical procedures are performed each year $(1,2)$. Consequently, surgery and anesthesia exposure have their own risks which can occur preoperatively, intraoperatively and postoperatively(3). While anesthesia related mortality and morbidity have fallen dramatically in recent decades (4), Post Operative Nausea and Vomiting (PONV) remains one of the most frequent and distressing complications following anesthetic and surgical procedures (5-7). It affects $20-30 \%$ of all post-operative surgical and $70 \%-80 \%$ high risk patients $(8,9)$.

Nausea is a subjective sensation of urge to vomit whereas vomiting as a clinical symptom is a forceful dynamic expulsion of gastric contents through the mouth or nose. Dry-retching is a vomit-like feelings 
with contractions of stomach without vomiting or substance (9). Post-operative nausea and vomiting (PONV) is a clinical manifestation that exist when a total score of nausea, dry retching and vomiting are greater than or equal to fifty depending on a Linear Post-operative Nausea and Vomiting Intensity Scale $(10,11)$. This activities are associated with increased heart rate, breathing and sweating (12). Although, it can occur in post-operative period during any time, the prevalence is large within $24 \mathrm{hrs}$. post operatively than after this hour (13).

It started as emesis from emetic center triggering by multiple factors which can be summarized as patient, anesthetic, and surgical factors. Patient background factors such as patients' age and sex, habits, motion sickness, history of PONV, full stomach and anxiety are important predictors of PONV. Anesthesia and surgery related factors such as use of volatile agents, type and duration of anesthesia, maintenance and reversal agents, and perioperative use of opioids are important predictors $(7,14)$.

Because PONV has a multi-factorial etiology, strategies to prevent and treat are also multi-factorial. They fall into: risk-reduction strategies, pharmacological interventions and non-pharmacological interventions $(13,15)$. Firstly, the risk reduction strategies management of PONV should involve preventing maneuvers that provoke PONV and universal multimodal pharmacologic prophylaxis (16).Secondly, pharmacological interventions such as, $\mathrm{H} 1$ and $\mathrm{H} 3$ antagonists, Steroids and Phenothiazine are advocated as treatment $(13,15)$. Lastly, acupuncture and acupressure has been postulated as non-pharmacological therapies prophylactic measure to prevent or reduce PONV (17).

Although the available antiemetic drugs used for prevention and management of PONV have been proven safe in clinical trials, no agent is without its side-effects. Some of the most commonly described side effects of these prophylaxis are headaches, hypotension, sedation, insomnia and QT interval prolongation. As a result, it has been advocated that antiemetic prophylactic regimens should be tailored to the patients most likely to experience PONV $(6,18)$.

Because of this, an identification of independent PONV risk factors would make it easier to select specific patients for effective antiemetic therapy (19).

\section{Methods And Materials}

Cross-sectional study was conducted at Jimma Medical Center from August 1, 2019 to September 30, 2019. The Source Population consisted of all adult elective surgical patients who were operated under anesthesia at Jimma Medical Center. Study Population included all adult elective surgical patients who were operated under anesthesia during the study period. Inclusion Criteria were: adult elective patients who were operated under general, spinal or combined GA and SA anesthesia and those who were wlling to give informed written consent. On the otherhand, operations that were performed in the outpatient department, patients who were unconscious postoperatively, patients who were re-operated, patients with medical and surgical conditions that could lead to nausea and vomiting, patients discharged before 24 hours postoperatively were excluded. The formula for calculating sample size (estimation of population proportion) is: 


$$
\mathrm{n}=\frac{(\mathrm{Z} \alpha / 2)^{2} \times \mathrm{p}(1-\mathrm{p})}{\mathrm{d}^{2}}=\frac{(1.96)^{2} \times 0.35(1-0.35)}{0.05^{2}}=350
$$

Where, $n=$ Sample size $=350, C . I=95 \%, a=0.05, D=$ Precision $=0.05 P=35 \%$ (prevalence of PONV from previous study at Gondar University Specialized Hospital), Non-respondent rate $(10 \%)=35$, Therefore, total sample size $=385$. Consecutive sampling technique was used. The study was conducted using a structured questionnaire and anesthetic record sheet for secondary data which was obtained from patient's chart and documentation. Linear PONV Intensity Scale was used to measure the incidence of PONV. It is believed to be a valid, reliable, and responsive measure of clinically important PONV. Accordingly, clinically important PONV was defined as a total score $\geq 50$ at any time throughout the study period (20). The intensity of pain was evaluated using verbal rating scale. It is a subjective measure in which individuals rate their pain as no pain; mild pain; moderate pain; and severe or intense pain as an average during the 24 hours assessment period. Assessment of pain was done by asking patients to rate their intensity of pain after surgery, by indicating which of the four words read aloud gave the best description of their pain (21). Lastly, to assess preoperative anxiety, the Amsterdam Preoperative Anxiety and Information Scale (APAl) was used. The Anxiety score is calculated as the sum of items 1, 2, 4 and 5 and the need for information score is the sum of items 3 and 6 . The score above 11 for the Anxiety subscale is a sign that the patient is experiencing anxiety (22). The study was conducted by interviewing patients using a structured questionnaire which were prepared in English and then translated into Amharic and Afan Oromo. Four data collectors were selected and one day training was given on how to complete data collection, and supervised by the investigator during data collection. In this study, count distinct episodes is defined as several vomits or retching events occurring over a short time frame, say 5 min, should be counted as one vomiting/dry-retching episode; multiple episodes require distinct time periods without vomiting/dry-retching. Clinically important PONV is defined as a total score $\geq 50$ at any time throughout the study period (20). Patients was asked by data collectors to self-report on demographic characteristics (sex, age, BMI) and patient related associated factors of PONV such as: previous history of motion sickness and migraine headache, smoking history and pre-operative anxiety. Other secondary data of intra-operative and post-operative factors such as, type of anesthesia, use of volatile anesthetics, intraoperative and post-operative use of opioids, duration and type of surgery, incidence of hypotension and use of reversal were obtained from the anesthetic record sheet, patients' chart, and operation registration books. The anxiety scale consists of four items (questions 1,2, 4, 5), each of which could be scored from 1 to 5 . The score of the anxiety scale is the sum of these four questions, with a scoring range from 4 to 20 . APAIS anxiety scale patients could be considered anxious at cutoff point of $\geq 11$ (22)

Training was given for data collectors. Then, the prepared format and questionnaire was given to data collectors. During data collection, regular supervisions and follow ups were made by the researcher and cross checked for completeness and consistency of collected data on daily basis. Once the data was collected and checked for completeness, consistency and accuracy, it was sorted and categorized. Then, the data entered into the computer using developed data entry format, coded for each category of 
variables and cross checked for errors. Statistical data analysis was done using SPSS software version 20. Data was summarized in form of proportions and frequency tables for categorical variables In the binary logistic regression analysis, Odds ratio together with $95 \%$ confidence interval was calculated to test for the association between the possible predictors and outcome variables (PONV). A p-value of less than 0.25 was considered as potential candidate for final model and entered into a multiple logistic regression to determine independent predictors of PONV. All variables that was found to be statistically significant ( $p$-value $<0.05$ ) on Multiple Logistic Regression analysis were considered as independent predictors of PONV.

\section{Results}

\section{Description of Socio demographic and Pre-operative Factors}

Out of a total of 385 adult patients, 368 included in the analysis (17 patients excluded due to incomplete documents and refusal) with response rate of $95 \%$. Of the total patients included in the analysis, $55.7 \%$ were female and $44.3 \%$ were male. The ages ranged from 20 to 88 years. Most of the patients (44\%) were aged between 20 and 30 years. The body mass index (BMI) was obtained by dividing weight (in kilograms) over square of the height (in meters). Forty (10.9\%) of the study patients were obese with a BMI of over 30. (Table 1).

Table :1 Summary of description of pre-operative factors among surgical elective patients at JUMC, from August 1, 2019 to September 30, 2019

\begin{tabular}{l|l|l|l}
\hline \multicolumn{2}{l|}{ Variables } & Frequency & Percentages \\
\hline \multirow{3}{*}{ Sex of patient } & Male & 163 & 44.3 \\
\cline { 2 - 4 } & Female & 205 & 55.7 \\
\hline \multirow{4}{*}{ BMI of the patient } & $20-30$ & 162 & 44 \\
\cline { 2 - 4 } & $31-40$ & 129 & 35.1 \\
\cline { 2 - 4 } & $41-50$ & 45 & 12.2 \\
\cline { 2 - 4 } & $>50$ & 32 & 8.7 \\
\cline { 2 - 4 } & $20-25$ (normal weight) & 224 & 12.0 \\
\cline { 2 - 4 } & $26-30$ (overweight) & 60 & 16.3 \\
\cline { 2 - 4 } & $>30$ (obese) & 40 & 10.9 \\
\hline
\end{tabular}

$(B M I=$ Body mass index,$P O N V=$ Post-operative nausea and vomiting $)$

\section{Peri-Operative Factors that are candidate for final mode}


Output of binary logistic regression analysis identifies the following pre-operative factors as potential candidate for final model (with $p$ value $<0.25$ ): Female gender, age, previous history of PONV and migraine headache, BMI and non administration of anti emetic prophylaxis (Table 2)

Table 2: Results of binary logistic regression analysis showing pre-operative factors that are candidate for final analysis model among surgical elective patients at JMC, from August 1, 2019 to September 30, 2019 


\begin{tabular}{|c|c|c|c|c|c|}
\hline \multirow[b]{2}{*}{ Variables } & & \multicolumn{2}{|c|}{ Incidence of PONV } & \multirow[b]{2}{*}{ COR $(95 \%$ CI) } & \multirow[b]{2}{*}{$\begin{array}{l}\mathrm{P}- \\
\text { value }\end{array}$} \\
\hline & & $\begin{array}{l}\text { Present } \\
(\%)\end{array}$ & $\begin{array}{l}\text { Absent } \\
(\%)\end{array}$ & & \\
\hline \multirow[t]{2}{*}{ Sex } & Male & $\begin{array}{l}18 \\
(11.0)\end{array}$ & $\begin{array}{l}145 \\
(89.0)\end{array}$ & 1 & \\
\hline & Female & $\begin{array}{l}83 \\
(40.5)\end{array}$ & $\begin{array}{l}122 \\
(59.5)\end{array}$ & $\begin{array}{l}5.480 \quad(3.119- \\
9.629) *\end{array}$ & 0.000 \\
\hline \multirow[t]{4}{*}{ Age } & $20-30$ & $\begin{array}{l}38 \\
(23.5)\end{array}$ & $\begin{array}{l}124 \\
(76.5)\end{array}$ & $\begin{array}{l}0.448 *(0.203- \\
0.990) *\end{array}$ & 0.047 \\
\hline & $31-40$ & $\begin{array}{l}30 \\
(23.3)\end{array}$ & $\begin{array}{l}99 \\
(76.7)\end{array}$ & $\begin{array}{ll}0.443 & (0.196- \\
1.001) & \end{array}$ & 0.050 \\
\hline & $41-50$ & $\begin{array}{l}20 \\
(44.4)\end{array}$ & $\begin{array}{l}25 \\
(55.6)\end{array}$ & $\begin{array}{l}1.169 \quad(0.467- \\
2.929) \quad\end{array}$ & 0.739 \\
\hline & $>50$ & $\begin{array}{l}13 \\
(40.6)\end{array}$ & $\begin{array}{l}19 \\
(59.4)\end{array}$ & 1 & \\
\hline \multirow[t]{4}{*}{$\begin{array}{l}\text { BMI } \\
\text { of the patient }\end{array}$} & $<20$ & 7 (15.9) & $\begin{array}{l}37 \\
(84.1) \\
\end{array}$ & 1 & \\
\hline & $20-25$ & $\begin{array}{l}59 \\
(26.3)\end{array}$ & $\begin{array}{l}165 \\
(73.7)\end{array}$ & $\begin{array}{lll}1.890 & (0.799 & - \\
4.470) & & \end{array}$ & 0.147 \\
\hline & $26-30$ & $\begin{array}{l}19 \\
(31.7)\end{array}$ & $\begin{array}{l}41 \\
(68.3)\end{array}$ & $\begin{array}{lll}2.449 & (0.925 & - \\
6.487) & & \end{array}$ & 0.071 \\
\hline & $>30$ & $\begin{array}{l}16 \\
(40.0)\end{array}$ & $\begin{array}{l}24 \\
(60.0)\end{array}$ & $\begin{array}{l}3.524(1.263- \\
9.831) *\end{array}$ & 0.016 \\
\hline \multirow[t]{2}{*}{$\begin{array}{l}\text { Previous history } \\
\text { of PONV }\end{array}$} & Yes & $\begin{array}{l}57 \\
(42.9)\end{array}$ & $\begin{array}{l}76 \\
(57.1)\end{array}$ & $\begin{array}{l}3.256 \\
5.234) *\end{array}$ & 0.000 \\
\hline & No & $\begin{array}{l}44 \\
(18.7)\end{array}$ & $\begin{array}{l}191 \\
(81.3)\end{array}$ & 1 & \\
\hline \multirow[t]{2}{*}{ Smoking history } & Smoker & $\begin{array}{l}12 \\
(32.4)\end{array}$ & $\begin{array}{l}25 \\
(67.8)\end{array}$ & 1 & \\
\hline & $\begin{array}{l}\text { Non } \\
\text { smoker }\end{array}$ & $\begin{array}{l}89 \\
(26.9)\end{array}$ & $\begin{array}{l}242 \\
(73.1)\end{array}$ & $\begin{array}{l}0.766 \quad(0.369- \\
1.590) *\end{array}$ & 0.024 \\
\hline \multirow[t]{2}{*}{$\begin{array}{l}\text { Previous history of migraine } \\
\text { headache }\end{array}$} & Yes & $\begin{array}{l}56 \\
(31.8)\end{array}$ & $\begin{array}{l}120 \\
(68.2)\end{array}$ & $\begin{array}{l}.524 \quad(0.962 \quad- \\
2.416) *\end{array}$ & 0.073 \\
\hline & No & $\begin{array}{l}45 \\
(23.4) \\
\end{array}$ & $\begin{array}{l}147 \\
(76.6) \\
\end{array}$ & 1 & \\
\hline \multirow[t]{2}{*}{$\begin{array}{l}\text { Administration of antiemetic } \\
\text { prophylaxis }\end{array}$} & Yes & $\begin{array}{l}12 \\
(14.0)\end{array}$ & $\begin{array}{l}74 \\
(86.0)\end{array}$ & 1 & \\
\hline & No & 89 & 193 & $2.844 \quad(1.470-$ & 0.002 \\
\hline
\end{tabular}




\begin{tabular}{l|l|l|l|l|l|l} 
& & $(31.6)$ & $(68.4)$ & $5.500) *$ & \\
\hline Presence of anxiety & Yes & $\begin{array}{l}35 \\
(34.0)\end{array}$ & $\begin{array}{l}68 \\
(66.0)\end{array}$ & $\begin{array}{l}1.552 \quad(0.947 \quad-543) * \\
0.810\end{array}$ & \\
\cline { 2 - 7 } & & No & 66 & 199 & 1 & \\
& & $(24.9)$ & $(75.1)$ & & \\
\hline
\end{tabular}

(PONV - Post-operative nausea and vomiting, 1 - Reference category, COR - Crude Odds Ratio, CI - Confidence Interval, * - significantly associated variables that are candidate for final analysis model at $P$ value less than 0.25

\section{Intra-operative factors that were potential candidate for final model}

Intra-operative factors that were potential candidate for final model (with $p$ value $<0.25$ ) after binary logistic regression analysis included type of anesthesia used, use of intra-operative opioids, type of surgery, duration of anesthesia, administration of neostigmine and incidence of intraoperative hypotension. Conversely, type of induction and maintenance agents were not candidate for final model after binary logistic regression analysis(Table 3).

Table 3: Results of binary logistic regression analysis showing intra-operative factors that are candidate for final analysis model among surgical elective patients at JMC, from August 1, 2019 to September 30, 2019 


\begin{tabular}{|c|c|c|c|c|c|}
\hline \multirow[t]{2}{*}{ Variables } & & \multicolumn{2}{|c|}{ Incidence of PONV } & \multirow{2}{*}{ COR (95\% CI) } & \multirow[b]{2}{*}{$\begin{array}{l}\mathrm{P}- \\
\text { vale }\end{array}$} \\
\hline & & $\begin{array}{l}\text { Present } \\
(\%)\end{array}$ & $\begin{array}{l}\text { Absent } \\
(\%)\end{array}$ & & \\
\hline \multirow[t]{4}{*}{ Types of anesthesia used } & GA with ETT & $\begin{array}{l}59 \\
(35.5)\end{array}$ & $\begin{array}{l}107 \\
(64.5)\end{array}$ & $\begin{array}{ll}0.551 & (0.240- \\
1.267) & \\
\end{array}$ & 0.161 \\
\hline & GA sedation & $6(16.7)$ & $\begin{array}{l}30 \\
(83.3)\end{array}$ & $\begin{array}{l}0.200 *(0.062- \\
0.642) *\end{array}$ & 0.007 \\
\hline & SA & $\begin{array}{l}23 \\
(16.4)\end{array}$ & $\begin{array}{l}117 \\
(83.6)\end{array}$ & $\begin{array}{l}0.197 *(0.081- \\
0.478) *\end{array}$ & 0.000 \\
\hline & GA and SA & $\begin{array}{l}13 \\
(50.0)\end{array}$ & $\begin{array}{l}13 \\
(50.0)\end{array}$ & 1 & \\
\hline \multirow[t]{4}{*}{$\begin{array}{l}\text { Type of induction agent } \\
\text { for GA }\end{array}$} & Ketamine & $\begin{array}{l}38 \\
(39.6) \\
\end{array}$ & $\begin{array}{l}58 \\
(60.4) \\
\end{array}$ & 1 & \\
\hline & Thiopentone & $\begin{array}{l}14 \\
(34.1)\end{array}$ & $\begin{array}{l}27 \\
(65.9) \\
\end{array}$ & $\begin{array}{ll}0.791 & (0.369 \\
-1.699) & \\
\end{array}$ & 0.618 \\
\hline & Propofol & $\begin{array}{l}20 \\
(26.3)\end{array}$ & $\begin{array}{l}56 \\
(73.7)\end{array}$ & $\begin{array}{ll}0.545 & (0.283 \\
-1.049) & \\
\end{array}$ & 0.952 \\
\hline & Combination & $6(33.3)$ & $\begin{array}{l}12 \\
(66.7)\end{array}$ & $\begin{array}{ll}0.763 & (0.264 \\
-2.207) & \\
\end{array}$ & 0.551 \\
\hline \multirow[t]{2}{*}{ Agent for maintenance } & Inhal. agents & $\begin{array}{l}60 \\
(33.0) \\
\end{array}$ & $\begin{array}{l}122 \\
(67.0)\end{array}$ & $\begin{array}{lll}1.059 & (0.512 & - \\
2.191) & & \\
\end{array}$ & 0.877 \\
\hline & IV agents & $\begin{array}{l}13 \\
(31.7)\end{array}$ & $\begin{array}{l}28 \\
(68.3)\end{array}$ & 1 & \\
\hline \multirow[t]{2}{*}{ Intraoperative opioids } & Used & $\begin{array}{l}75 \\
(34.6) \\
\end{array}$ & $\begin{array}{l}142 \\
(65.4)\end{array}$ & $\begin{array}{l}2.336 \quad(1.404- \\
3.888) * \\
\end{array}$ & 0.001 \\
\hline & Not used & $\begin{array}{l}26 \\
(18.4)\end{array}$ & $\begin{array}{l}115 \\
(81.6)\end{array}$ & 1 & \\
\hline \multirow[t]{2}{*}{ Use of neostigmine } & Used & $\begin{array}{l}53 \\
(39.0) \\
\end{array}$ & $\begin{array}{l}83 \\
(61.0)\end{array}$ & $\begin{array}{l}2.554 \quad(1.585 \\
4.117) * \\
\end{array}$ & 0.000 \\
\hline & Not used & $\begin{array}{l}44 \\
(20.0)\end{array}$ & $\begin{array}{l}176 \\
(80.0)\end{array}$ & 1 & \\
\hline \multirow[t]{2}{*}{$\begin{array}{l}\text { Intraoperative } \\
\text { hypotension }\end{array}$} & Present & $\begin{array}{l}18 \\
(43.9) \\
\end{array}$ & $\begin{array}{l}23 \\
(56.1) \\
\end{array}$ & $\begin{array}{l}2.301(1.183- \\
4.474) *\end{array}$ & 0.014 \\
\hline & Not present & $\begin{array}{l}83 \\
(25.4) \\
\end{array}$ & $\begin{array}{l}244 \\
(74.6) \\
\end{array}$ & 1 & \\
\hline \multirow[t]{2}{*}{$\begin{array}{l}\text { Type of } \\
\text { performed }\end{array}$} & Thyroid & $4(20.0)$ & $\begin{array}{l}16 \\
(80.0) \\
\end{array}$ & $\begin{array}{ll}1.750 & (0.384 \\
-7.969) & \\
\end{array}$ & 0.469 \\
\hline & Gynecological & $68(46.3)$ & $79(53.7)$ & $6.025 \quad(2.012 \quad-$ & 0.001 \\
\hline
\end{tabular}




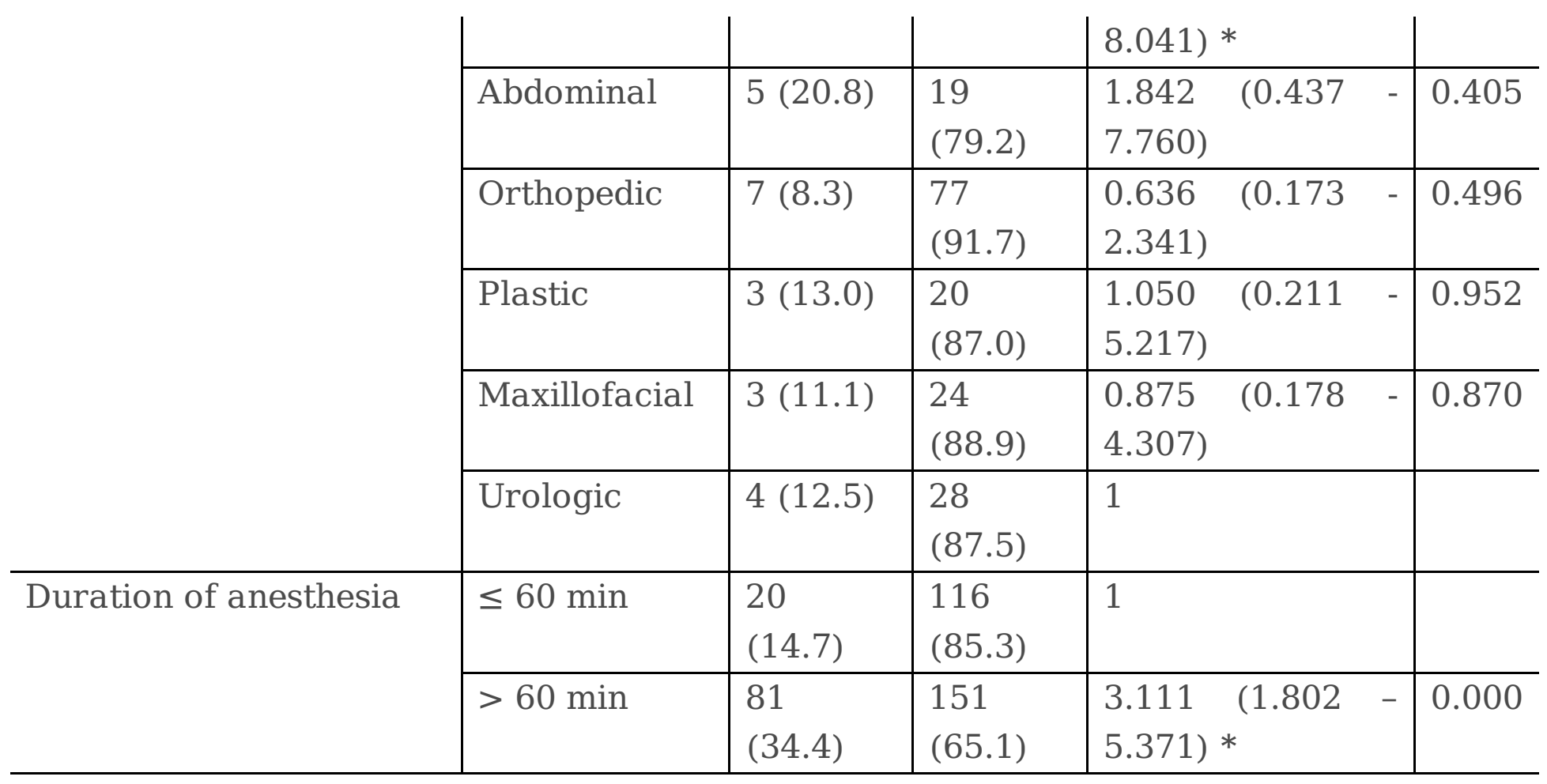

(PONV - Post-operative nausea and vomiting, 1 - Reference category, COR - Crude Odds Ratio, CI - Confidence Interval, GA - General anesthesia, SA - Spinal anesthesia, IV intravenous anesthesia, ETT - Endotracheal intubation, * - significantly associated variables that are candidate for final analysis model at $P$ value less than 0.25 )

\section{Post-operative factors that were potential candidate for final model}

Post-operative factors such as, administration of post-operative opioid and severity of post-operative pain were identified as potential candidate for multiple logistic regression. However, time of first oral intake was not candidate for final model (Table 4)

Table 4: Results of binary logistic regression analysis showing post-operative factors that are candidate for final analysis model among surgical elective patients at JMC, from August 1, 2019 to September 30, 2019 


\begin{tabular}{|c|c|c|c|c|c|}
\hline \multirow{2}{*}{\multicolumn{2}{|c|}{ Variables }} & \multicolumn{2}{|c|}{ Incidence of PONV } & \multirow[b]{2}{*}{ COR $(95 \% \mathrm{CI})$} & \multirow{3}{*}{$\begin{array}{l}\mathrm{P} \text { - } \\
\text { value }\end{array}$} \\
\hline & & $\begin{array}{l}\text { Present } \\
(\%)\end{array}$ & $\begin{array}{l}\text { Absent } \\
(\%)\end{array}$ & & \\
\hline \multirow[t]{4}{*}{$\begin{array}{l}\text { Timing of first oral intake after } \\
\text { operation }\end{array}$} & $0-6$ & $6(27.3)$ & $\begin{array}{l}16 \\
(72.7)\end{array}$ & 1 & \\
\hline & $7-12$ & $8(15.4)$ & $\begin{array}{l}44 \\
(84.6)\end{array}$ & $\begin{array}{lll}0.485 & (0.146 \quad- \\
1.615) & & \\
\end{array}$ & 0.288 \\
\hline & $13-24$ & $\begin{array}{l}53 \\
(27.6)\end{array}$ & $\begin{array}{l}139 \\
(72.4)\end{array}$ & $\begin{array}{lll}1.017 & (0.378 \\
2.737) & \end{array}$ & 0.974 \\
\hline & $>24$ & $\begin{array}{l}34 \\
(33.3) \\
\end{array}$ & $\begin{array}{l}68 \\
(66.7) \\
\end{array}$ & $\begin{array}{lll}1.333 & (0.479 \quad- \\
3.715) & & \\
\end{array}$ & 0.583 \\
\hline \multirow[t]{2}{*}{ Severity of post-operative pain } & $\begin{array}{l}\text { Less } \\
\text { pain }\end{array}$ & $\begin{array}{l}32 \\
(19.2)\end{array}$ & $\begin{array}{l}135 \\
(80.8)\end{array}$ & 1 & \\
\hline & $\begin{array}{l}\text { More } \\
\text { pain }\end{array}$ & $\begin{array}{l}69 \\
(34.3)\end{array}$ & $\begin{array}{l}132 \\
(65.7)\end{array}$ & $\begin{array}{l}2.205(1.361- \\
3.575) *\end{array}$ & 0.001 \\
\hline \multirow[t]{2}{*}{ Post-operative use of opioids } & Used & $\begin{array}{l}77 \\
(37.7) \\
\end{array}$ & $\begin{array}{l}127 \\
(62.3)\end{array}$ & $\begin{array}{l}3.537(2.108- \\
5.933 *\end{array}$ & \\
\hline & $\begin{array}{l}\text { Not } \\
\text { used }\end{array}$ & $\begin{array}{l}24 \\
(14.6)\end{array}$ & $\begin{array}{l}140 \\
(85.4)\end{array}$ & 1 & 0.000 \\
\hline
\end{tabular}

(PONV - Post-operative nausea and vomiting, 1 - Reference category, COR - Crude Odds Ratio, CI - Confidence Interval, * - significantly associated variables that are candidate for final analysis model at $P$ value less than 0.25 )

\subsection{Independent Predictors of PONV}

Risk factors associatedwith PONV were analyzed further. All factors that turned out with a significant level ( $p$ value $<0.25$ ) on after binary logistic regression analysis were used to model for final analysis. Consequently, backward multiple logistic regression analysis was used to determine the association of perioperative factors with the incidence of PONV. Accordingly, output of multiple logistic regression model revealed that sex, history of PONV or motion sickness, long duration of anaesthesia, Gynecological type of surgery and administration of post-operative opioids as independent predictors of PONV at significant level $(p<0.05)$.

Female patients was identified as strongest pre-operative independent predictor with $(\mathrm{AOR}=4.065, \mathrm{Cl}=$ 2.090 - 7.906). Whereas, previous history of PONV or motion sickness was also one of independent predictor of PONV with $(A O R=2.836, \mathrm{Cl}=1.582-5.083)$. Among intra-operative factors, only duration of anesthesia greater than sixty minutes $(A O R=2.974, C l=1.491-5.933)$ and Gynecologic type of surgery 
$(\mathrm{AOR}=3.782, \mathrm{Cl}=1.156-12.373)$ were identified as independent predictor. Whereas, only post-operative opioids administration $(\mathrm{AOR}=2.333, \mathrm{Cl}=1.221-4.457)$ was identified as independent factors among post-operative factors (Table 5)

Table 5: Output of multiple logistic regression analysis model showing independent predictors of PONV among surgical elective patients at JMC, from August 1, 2019 to September 30, 2019

\begin{tabular}{|c|c|c|c|c|c|}
\hline \multicolumn{2}{|l|}{ Variables } & \multicolumn{2}{|c|}{ Incidence of PONV } & \multirow{2}{*}{ AOR $(95 \% \mathrm{CI})$} & \multirow{2}{*}{$\begin{array}{l}\mathrm{P}- \\
\text { value }\end{array}$} \\
\hline \multirow{3}{*}{ Sex } & Male & \multirow{2}{*}{$\begin{array}{c}\begin{array}{c}\text { Present } \\
(\%)\end{array} \\
18(11.0)\end{array}$} & 145 & & \\
\hline & & & $(89.0)$ & & \\
\hline & Female & $83(40.5)$ & $\begin{array}{l}122 \\
(59.5)\end{array}$ & $\begin{array}{l}4.065 \\
7.906) *\end{array}$ & 0.000 \\
\hline \multirow[t]{2}{*}{ History of PONV } & Yes & $57(42.9)$ & $76(57.1)$ & $\begin{array}{lll}2.836 \\
5.083) *\end{array}$ & 0.000 \\
\hline & No & $44(18.7)$ & $\begin{array}{l}191 \\
(81.3)\end{array}$ & 1 & \\
\hline \multirow[t]{2}{*}{ Duration of Anesthesia } & $\leq 60 \min$ & $20(14.7)$ & $\begin{array}{l}116 \\
(85.3)\end{array}$ & 1 & 0.002 \\
\hline & $>60 \mathrm{~min}$ & $81(34.4)$ & $\begin{array}{l}151 \\
(65.1)\end{array}$ & $\begin{array}{lll}3.089 & (1.467 & - \\
6.505) * & & \end{array}$ & \\
\hline \multirow[t]{2}{*}{ Post-operative opioids } & Used & $77(37.7)$ & $\begin{array}{l}127 \\
(62.3)\end{array}$ & $\begin{array}{l}2.333 \\
4.457) *(1.221\end{array}$ & 0.010 \\
\hline & Not used & $24(14.6)$ & $\begin{array}{l}140 \\
(85.4)\end{array}$ & 1 & \\
\hline \multirow{7}{*}{$\begin{array}{l}\text { Type of } \\
\text { performed }\end{array}$} & Thyroid & $4(20.0)$ & $16(80.0)$ & $\begin{array}{ll}0.835 & (0.161 \\
4.323) & \end{array}$ & \\
\hline & $\begin{array}{l}\text { Gynecology } \\
\text { gical }\end{array}$ & $68(46.3)$ & $79(53.7)$ & $\begin{array}{l}3.782(1.156 \\
12.373) *\end{array}$ & 0.028 \\
\hline & Abdominal & $5(20.8)$ & $19(79.2)$ & $\begin{array}{ll}3.133 \\
15.531)\end{array} \quad(0.632$ & \\
\hline & Orthopedic & $7(8.3)$ & $77(91.7)$ & $\begin{array}{lrll}0 & .460 \\
1.859) & (0.114 & \\
\end{array}$ & \\
\hline & Plastic & $3(13.0)$ & $20(87.0)$ & $\begin{array}{ll}1.677 & (0.298 \\
9.423) & \end{array}$ & \\
\hline & Maxillofacial & $3(11.1)$ & $24(88.9)$ & $\begin{array}{ll}0 & .688 \\
3.789) & (0.125\end{array}$ & \\
\hline & Urologic & $4(12.5)$ & $28(87.5)$ & 1 & \\
\hline
\end{tabular}

(PONV - Post-operative nausea and vomiting, 1 - Reference category, AOR - Adjusted Odds Ratio, COR - Crude Odds Ratio CI - Confidence Interval, * - independent predictors of PONV at $P$ value less than 0.05 )

\section{Discussion}


This study was designed to investigate the incidence of postoperative nausea and vomiting to ascertain the degree of discomfort after everyday surgery with modern anaesthetic and surgical techniques. Our finding has shown that the incidence of PONV is $27.4 \%$ at JMC, which indicate that the occurrence of PONV in this setup is high. Out of 368 study patients, 267 (72.6\%) were free of the complication. These findings are in line with studies done in South Africa which showed the incidence of PONV among African groups was $27 \%$ (23). This does not differ much from what studies done in Malawi which showed the incidence of PONV was $29 \%$ (24).

This finding was low when compared with other studies done in Africa and Asia. Consequently, different studies conducted in Nigeria (25), Uganda (26), Tanzania (27) and Ghana (28), found the incidence of PONV 41\%, 40.7\%, 41.4\%, and 34\% respectively. In India (7) and Singapore (29) it was found to be $33.3 \%$ and $34.1 \%$, respectively. This might be due to techniques used in their studies to measure PONV have included traditional methods (absolute counts, Likert scales, visual analogue scales (VAS), and treatment response). They just defined PONV as having at least one episode of nausea, vomiting or both within 24 hours post-operatively. Due to this they might be included PONV condition which was not clinically significant. However, this study has used a recently developed PONV Intensity Scale, which is believed to be a valid, reliable, and responsive measure of clinically important PONV (20). On the other hand, this study finding is high when compared with the studies in developed countries. A recent study in South Korea by J.Choi found the incidence of PONV was 23\% (30). Similarly, in Turkey it was found to be $25 \%$ (31). This difference in incidence might be due to anesthetic and surgical techniques differences (9) and study design.

In this study, the incidence of PONV were higher in the female patients. Among the females, $40.5 \%$ had PONV compared to $11 \%$ in males. The female gender increased the likelihood of PONV by four times and it was the strongest predictor of PONV with AOR of 4.065 (2.090 - 7.906). This finding was consistent with what has previously been reported from studies done elsewhere. Morino, in Japan found that women had significantly more likely to develop PONV (four times) than men and suggested that women be considered for prophylactic anti-emetic therapy (32). Similarly, N. Rodseth, in South Africa found that women patients were more likely to develop PONV than men and suggested for prophylactic anti-emetic therapy (23). A descriptive study conducted at Toronto Hospital, Canada, found that women had a nearly two fold higher rate of PONV than male (33). In the same way, a 2012 systematic review study of evidence based analysis risk factor of PONV from San Francisco (USA), identified being a female as strongest patient specific predictor out of the eight patient-specific risk factors (34). This is due to vomiting center in females is influenced by the endocrine or hormonal environment (35).

A previous history of PONV or motion sickness was also strongly associated with PONV. Among the seventeen six study patients who had PONV previously, $42.9 \%$ had incidence of PONV while only $18.7 \%$ had developed in group with no history of PONV. This group of patients was three times more likely to have PONV with AOR of 2.836 (1.582 - 5.083). Similar findings have been noted in other studies. This finding is similar to the study done by Morino, C. C. Apfel (32) and N. Rodseth, in South Africa (23). In these studies, previous history of PONV or motion sickness was a strong risk factor with AOR of 3.65 
(2.52-5.30) and $2.6(1.8-3.7)$, respectively. Similarly, it was the second strongest predictor of PONV in a Korean Predictive Model for Postoperative Nausea and Vomiting study with odds ratio of 2.4 (36). This is because of genetic factors determine sensitivity to motion sickness(37) and because individuals with a history of these factors have already established reflex arch for vomiting $(9,38)$. Other pre-operative factors such as, age of patient, BMI, pre-operative anxiety, history of migraine headache and smoking history is reported to affect the prevalence of PONV, but these factors were not found to be independent predictors of PONV after multiple logistic regression in this study.

Consequently, duration of anesthesia was one of intra-operative independent predictors of PONV. It was found patients who had duration of anesthesia greater than sixty minutes were three times more likely developed PONV in comparison than those duration of less than sixty minutes with adjusted odds ratio of 2.974 (1.491 - 5.933). This result was in agreement with what has previously been reported from different studies $(8,33,34)$. One study indicated that for every 30 -minute increase in surgical time, there was a $59 \%$ rise in the baseline risk of PONV (33). Similarly, in a Korean Predictive Model for Postoperative Nausea and Vomiting study, duration of anesthesia was a predictor of PONV with the odds ratio of 1.9 for the anesthesia time longer than 1 hour (36). This is due to prolonged surgery increases exposure to anesthetic agents, use of intraoperative opioids, and increased exposure to hemodynamic instability which increases incidence of PONV (9).

Output of multiple logistic regression analysis also revealed that among type of surgery only Gynecologic surgery was significantly associated with PONV as independent predictor of PONV. In patients who underwent Gynecologic surgery the incidence of PONV was four times more likely occurred than those with non-Gynecologic surgeries with AOR of 3.782 (1.156 -12.373). This result was in agreement with what has previously been reported from other studies. In a 2012 cross-sectional study at the university of Gondar teaching hospital, northwest, Ethiopia, Gynaecology operation was identified as independent predictor of PONV with AOR of 2.58 (1.24-5.39)(39). This might be due to stimulation of vagal afferents nerves during gynecologic surgery manipulation that result in activation of vomiting center $(4,48)$. Furthermore, it might be because of patients are all females (34). In contrast to this, a 2012 systematic review done by Apfel, and his co-workers, were they stated that "there is no or insufficient evidence for type of surgery to be an independent risk factor for PONV (34). This is possibly due to differences in study design and study population. On the other hand, despite intra-operative factors such as, type of anesthesia, incidence of intra-operative hypotension, administration of neostigmine and intra-operative use of opioids were included in final model, they were not independent predictors of PONV after multiple logistic regression.

Post-operative administration of opioid was the only post-operative factor that was identified as independent predictor of PONV after multiple logistic regression. Among 204 study patients who had taken post-operative opioids, $37.7 \%$ had PONV compared to $14.6 \%$ of those who hadn't taken. In patients who had received post-operative opioids, the incidence of PONV was two times more likely developed when compared with those who hadn't received post-operative opioids with AOR of 2.333 (1.221 - 4.457). This was in line with findings of studies done elsewhere. Roberts et al, in Australia found postoperative 
opioid use to significantly $(p=0.025)$ affect the incidence of PONV, stating that post-operative opioid administration increases the incidence of PONV in dose dependent manner(40). Similarly, in a 2012 systematic review study of evidence based analysis risk factor of PONV from San Francisco (USA), it was the strongest anaesthesia-related predictor of PONV(34). The reason for this is due to the fact that opioids directly stimulates the Chemoreceptor Trigger Zone and vestibular apparatus, and also decreases motility of the gut increasing the incidence of PONV (4).

The strength of this study was that we have used a recently developed technique (linear PONV Intensity Scale), a reliable technique which is used to identify clinically significant PONV. Limitations of the Study include some peri-operative factors were not well documented

\section{Conclusion}

The overall incidence of post-operative nausea and vomiting in the surgical patients at Jimma Medical Center is high. Female patients, patients with previous history of PONV or motion sickness, long duration of anaesthesia, patients who underwent Gynecologic type of surgery and received post-operative opioids.

\section{Declarations}

Ethical approval and consent to participate

Our study did not involve human participants, human data or human tissue as our study is observatih'. Letter of permission and ethical approval for the study was obtained from Ethical Review Board of JUIH. Written informed consent were taken from all the study patients. Information obtained was used only for study purposes and every information was kept confidential for privacy of the patient.

Consent for publication: Not applicable

Availability of data and materials: Not applicable

Competing interests: We declared that we had no competing interests.

Funding: Jimma University.

Competing interest: the authors declared that they have no conflict of interest.

Author's contribution: DK initiated the research, wrote the research proposal, conducted the search, did data entry and analysis and wrote the manuscript MS,ND, GT,ZM and SM Initiated the research, wrote the research proposal, conducted the research, did data entry and analysis and wrote the manuscript. All authors read and approve the final manuscript.

Acknowledgement: We would like to acknowledge Jimma University for providing materials used for the work. 


\section{References}

1. Weiser TG, Haynes AB, Molina G, Lipsitz SR, Esquivel MM, Uribe-leitz T, et al. Size and distribution of the global volume of surgery in 2012 Global volume of surgery. 2016;i(March):201-9.

2. Weiser TG, Haynes AB, Molina G, Lipsitz SR, Esquivel MM, Uribe-leitz T, et al. Surgical Services: Access and Coverage Estimate of the global volume of surgery in 2012: an. 2012;94305.

3. Merry AF, Mitchell SJ. Complications of anaesthesia. 2018;73:7-11.

4. John Doyle D, Dahaba A, LeManach Y. Advances in anesthesia technology are improving patient care, but many challenges remain. BMC Anesthesiol. 2018;18(1):1-5.

5. For PM, Factor R, Ponv P. PONV Prophylaxis Guidelines. 2015;(October):1-4.

6. Gan TJ, Diemunsch P, Habib AS, Kovac A, Kranke P, Meyer TA, et al. Consensus Guidelines for the Management of Postoperative Nausea and Vomiting. 2014;118(1):85-113.

7. Manoharan AK, Mallick NA, Thangavelu S. A Cross Sectional Study on Postoperative Nausea and Vomiting-Risk Prediction and Assessment in Patients Undergoing Elective Surgical Patients. 2017;4(3):640-4.

8. Srilata M, Jayaram K. Nausea and Vomiting. 2016;345-57.

9. Balaban CD, Yates BJ. Autonomic Neuroscience: Basic and Clinical What is nausea? A historical analysis of changing views. Auton Neurosci Basic Clin. 2017;202:5-17.

10. Myles PS, Wengritzky R. Simplified postoperative nausea and vomiting impact scale for audit and post-discharge review. 2012;108(January):423-9.

11. Wengritzky R, Mettho T, Myles PS, Burke J, Kakos A. Development and validation of a postoperative nausea and vomiting intensity scale. Br J Anaesth. 2010;104(2):158-66.

12. Golembiewski J. Antiemetics: Focus on Pharmacology. J PeriAnesthesia Nurs. 2014;29(6):514-8.

13. Blackburn J. Postoperative nausea and vomiting. Anaesth Intensive Care Med. 2015;16(9):452-6.

14. Parra-sanchez I, Abdallah R, You J, Fu AZ, Grady M, lii KC, et al. A time-motion economic analysis of postoperative nausea and vomiting in ambulatory surgery ' conomique des temps et mouvements pour les Une analyse e ' es et vomissements postope ' ratoires en chirurgie d ' un jour nause. 2012;366-75.

15. Whelan R. Nausea and vomiting after surgery. 2018;13(1):28-32.

16. Views E. me Cost-eflective Management of Postoperative Nausea and Vomiting. 2019;(4):4-6.

17. Noroozinia H, Mahoori A, Hasani E. The Effect of Acupressure on Nausea and Vomiting after Cesarean Section Under Spinal Anesthesia. 2013;(9).

18. Obrink E, Jildenstål P, Oddby E, Jakobsson JG. Post-operative nausea and vomiting: Update on predicting the probability and ways to minimize its occurrence, with focus on ambulatory surgery. 2015;15:100-6.

19. Gan TJ, Diemunsch P, Habib AS, Kovac A, Kranke P, Meyer TA, et al. Consensus guidelines for the management of postoperative nausea and vomiting. Anesth Analg. 2014;118(1):85-113. 
20. Wengritzky R, Mettho T, Myles PS, Burke J, Kakos A. CLINICAL PRACTICE Development and validation of a postoperative nausea and vomiting intensity scale. 2010;104(2):158-66.

21. Williamson A, Hoggart B. Pain: a review of three commonly used pain rating scales. 2005;1994(Spence 2000):798-804.

22. Moerman N, Oosting H. The Amsterdam Scale (APAIS). 1996;

23. Rodseth RN, Gopalan PD, Cassimjee HM, Goga S. Reduced incidence of postoperative nausea and vomiting in black south africans and its utility for a modified risk scoring system. Anesth Analg. 2010;110(6):1591-4.

24. Mndolo S, Jung K, Pollach G. Postoperative Nausea \& Vomiting in Malawi. 2014;(April):447-51.

25. Nze P. A survey of postoperative nausea and vomiting in Enugu, Nigeria. Orient $\mathrm{J}$ Med. $2011 ; 17(3): 443-9$.

26. Ssebuufu R, Kakande I, Okello M. Post-operative Nausea and Vomiting at Mulago Hospital. East Cent Afr.j.surg(online). 2009;14(2):50-7.

27. Sciences-bugando A. Postoperative nausea and vomiting at a tertiary care hospital in north- western Tanzania. 2015;17(3):1-9.

28. Amponsah G. POSTOPERATIVE NAUSEA AND VOMITING IN KORLE BU TEACH- ING HOSPITAL. 2007; (December):181-5.

29. Yu X, Leong A, Wen X, Kwa C, Hui S, Ng E, et al. Singapore General Hospital Experience on Ethnicity and the Incidence of Postoperative Nausea and Vomiting after Elective Orthopaedic Surgeries. $2015 ; 2015$.

30. Choi JB, Shim YH, Lee Y, Lee JS, Choi J, Chang CH. Incidence and Risk Factors of Postoperative Nausea and Vomiting in Patients with Fentanyl-Based Intravenous Patient-Controlled Analgesia and Single Antiemetic Prophylaxis. 2019;55(5):1430-5.

31. Article O. Risk Factors for Postoperative Nausea and Vomiting in Pediatric Patients Undergoing Ambulatory Dental Treatment. 2018;597-602.

32. Morino R, Ozaki M, Nagata O. Incidence of and risk factors for postoperative nausea and vomiting at a Japanese Cancer Center: first large-scale study in Japan. 2013;18-24.

33. Sinclair DR, Chung F, Mezei G, Ph D. Can Postoperative Nausea and Vomiting Be Predicted? 2019; (1):109-18.

34. Apfel CC, Heidrich FM, Jalota L, Hornuss C, Whelan RP, Zhang K, et al. Evidence-based analysis of risk factors for postoperative. Br J Anaesth. 2012;109(5):742-53.

35. Buchanan FF, Myles PS, Cicuttini F. Effect of patient sex on general anaesthesia and recovery. Br J Anaesth. 2011;106(6):832-9.

36. Duck HC, Justin SK, Hyun JA, Jie AK. A Korean predictive model for postoperative nausea and vomiting. J Korean Med Sci. 2005;20(5):811-5.

37. Horn CC, Meyers K, Oberlies N. Physiology \& Behavior Musk shrews selectively bred for motion sickness display increased anesthesia-induced vomiting. Physiol Behav. 2014;124:129-37. 
38. Murphy MJ, Hooper VD, Sullivan E, Clifford T, Apfel CC. Identification of Risk Factors for Postoperative Nausea and Vomiting in the Perianesthesia Adult Patient. 2006;

39. Prevalence and factors associated with post-operative nausea and vomiting at University of Gondar Hospital, Northwest Ethiopia, 2012: Across-sectional study. PREVALENCE AND FACTORS ASSOCIATED WITH POSTOPERATIVE NAUSEA AND VOMITING AT THE UNIVERSITY OF. 2015; (September 2014).

40. Roberts GW, Bekker TB, Carlsen HH, Moffatt CH, Slattery PJ, McClure F. Postoperative nausea and vomiting are strongly influenced by postoperative opioid use in dose-related a manner. Anesth Analg. 2005;101(5):1343-8. 